\title{
Kondo-enhanced Andreev tunneling in InAs nanowire quantum dots
}

Jespersen, Thomas Sand; Paaske, Jens; Andersen, Brian Møller; Grove-Rasmussen, Kasper; Jørgensen, Henrik Ingerslev; Aagesen, Martin; Sørensen, C. B.; Lindelof, Poul Erik; Flensberg, Karsten; Nygård, Jesper

Published in:

Physical Review Letters

Publication date:

2007

Document version

Early version, also known as pre-print

Citation for published version (APA):

Jespersen, T. S., Paaske, J., Andersen, B. M., Grove-Rasmussen, K., Jørgensen, H. I., Aagesen, M., Sørensen, C. B., Lindelof, P. E., Flensberg, K., \& Nygård, J. (2007). Kondo-enhanced Andreev tunneling in InAs nanowire quantum dots. Physical Review Letters, 99(12), 126603. 


\title{
Kondo-Enhanced Andreev Tunneling in InAs Nanowire Quantum Dots
}

\author{
T. Sand-Jespersen, J. Paaske, B. M. Andersen, K. Grove-Rasmussen, H. I. Jørgensen, M. Aagesen, C. B. Sørensen, \\ P. E. Lindelof, K. Flensberg, and J. Nygård \\ Nano-Science Center, Niels Bohr Institute, University of Copenhagen, Universitetsparken 5, DK-2100 Copenhagen, Denmark
} (Received 10 March 2007; published 19 September 2007)

\begin{abstract}
We report measurements of the nonlinear conductance of InAs nanowire quantum dots coupled to superconducting leads. We observe a clear alternation between odd and even occupation of the dot, with subgap peaks at $\left|V_{\mathrm{sd}}\right|=\Delta / e$ markedly stronger (weaker) than the quasiparticle tunneling peaks at $\left|V_{\mathrm{sd}}\right|=$ $2 \Delta / e$ for odd (even) occupation. We attribute the enhanced $\Delta$ peak to an interplay between Kondo correlations and Andreev tunneling in dots with an odd number of spins, and we substantiate this interpretation by a poor man's scaling analysis.
\end{abstract}

DOI: 10.1103/PhysRevLett.99.126603

PACS numbers: 72.15.Qm, 73.21.La, 73.23.-b

Since the discovery of the Kondo effect in quantum dots (QD) [1] this phenomenon has received extensive theoretical and experimental attention [2]. The effect emerges for QD's coupled strongly to the leads when the total spin of the electrons on the QD is nonzero, e.g., if it hosts an odd number of electrons $N$. At temperatures below the socalled Kondo temperature, $T_{K}$, the conduction electrons in the leads screen the spin through multiple cotunneling spin-flip processes resulting in a correlated many-body state which is experimentally observable as an increased linear conductance through the dot. If the leads consist of ( $s$-wave) superconductors (S) the conduction electrons form spin-singlet Cooper pairs incapable of flipping the dot spin, and therefore the Kondo effect and superconductivity constitute competing many-body effects.

Recent developments in techniques for fabrication of quantum dot systems have made it possible to produce $\mathrm{S}$ QD-S systems [3-7] enabling experimental studies of this intriguing interplay. In carbon nanotubes [3], it was found that the Kondo state persists when the energy needed for breaking the Cooper pairs is compensated by the energy gained in forming the Kondo state $\left(T_{K}>\Delta\right)$. Here $\Delta$ is the gap of the superconducting leads. Otherwise, the Kondo state is suppressed and the Kondo-induced increase in the linear conductance disappears.

For bias voltages less than $\left|V_{\mathrm{sd}}\right|=2 \Delta / e$, transport occurs through multiple Andreev reflection (AR) processes where electrons (holes) impinging on a superconducting electrode are reflected as holes (electrons) upon injecting (absorbing) a Cooper pair [8] as illustrated in Fig. 1(c) and $1(\mathrm{~d})$. The allowed processes depend on $V_{\text {sd }}$; upon increasing $\left|V_{\text {sd }}\right|$ through $2 \Delta / n e, n=2,3, \ldots$ processes containing $(n+1)$ AR are lost while those with $(n-1)$ AR start to contribute. This basic mechanism gives rise to a rich subharmonic gap structure (SGS) in electrical transport measurements which has been studied extensively in superconducting weak links [9], break junctions [10], and for quantum dots outside the Kondo regime [11]. Nevertheless, no experimental study of the influence of
Kondo correlations on the SGS has yet been reported and this is the focus of the present work. We find that even when the Kondo peak in the linear conductance is suppressed by the superconducting gap, a pronounced Kondo enhancement of the leading subgap peak in $d I / d V_{\mathrm{sd}}$ emerges at $\left|V_{\mathrm{sd}}\right|=\Delta / e$. We study the characteristics of this peak and attribute it to a Kondo enhancement of the Andreev-tunneling amplitude in dots with odd occupancy.
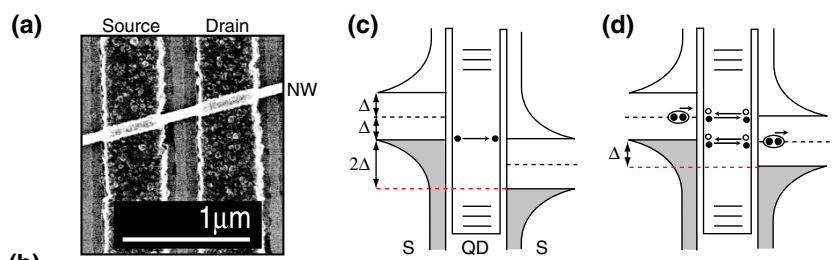

(b)

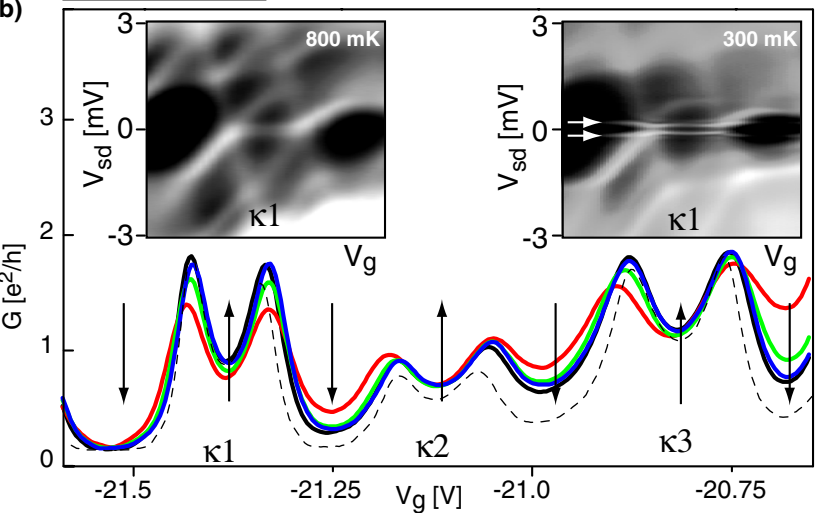

FIG. 1 (color online). (a) SEM image of a typical nanowire device. (b) Linear conductance in the Kondo regime for temperatures $\quad T=750 \mathrm{mK}($ black line $)-T=950 \mathrm{mK}($ red line $)$ and at $300 \mathrm{mK}$ (dashed line). The qualitative temperature dependence of the valley conductances for temperatures above $T_{c}$ are indicated by arrows. Leftmost inset shows $d I / d V_{\mathrm{sd}}$ vs $V_{\mathrm{sd}}$ and $V_{g}$ for the region of $\kappa_{1}$ at $800 \mathrm{mK}\left(>T_{c}\right)$. At $300 \mathrm{mK}$, i.e., below $T_{c}$ (rightmost inset) the Kondo ridge is suppressed. (c), (d) Schematic illustration of the processes which lead to peaks at $\left|V_{\mathrm{sd}}\right|=2 \Delta / e$ and $\left|V_{\mathrm{sd}}\right|=\Delta / e$, respectively. 
Our devices are based on semiconducting InAs nanowires grown by molecular beam epitaxy [12] and electrically contacted by a superconducting $\mathrm{Ti} / \mathrm{Al} / \mathrm{Ti}$ trilayer $(10 / 60 / 10 \mathrm{~nm})$ [4]. The superconducting transition temperature $T_{c} \approx 750 \mathrm{mK}$ and critical magnetic field $B_{c} \approx$ $250 \mathrm{mT}$ of the contacts are determined experimentally. Figure 1(a) shows a scanning electron micrograph of a device. The wire has a diameter $d \approx 70 \mathrm{~nm}$ and the electrode separation is $L \approx 300 \mathrm{~nm}$. As in our previous studies of nanowire devices contacted by (normal metal) $\mathrm{Ti} / \mathrm{Au}$ leads, tunnel barriers develop close to the nanowire-metal interfaces and due to spatial confinement of the wire, a quantum dot with discrete eigenstates is formed [13]. The barrier transparency and carrier concentration can be tuned by applying a voltage $V_{g}$ to the degenerately doped $\mathrm{Si}$ substrate, and below we focus on the regime appropriate for Kondo physics. The two-terminal conductance of the device is measured as a function of applied source-drain bias and gate potential using standard lock-in techniques.

We first characterize the device with the contacts in the normal state. Figure 1(b) shows the linear conductance $G$ as a function of $V_{g}$ for temperatures $750 \mathrm{mK}-950 \mathrm{mK}$ when the contacts are normal (solid lines). A series of overlapping Coulomb peaks are observed and the temperature dependence of the valley conductances are indicated by the arrows. In four valleys, the conductance decreases upon lowering the temperature, as expected for Coulomb blockade. For the three valleys labeled $\kappa_{1}-\kappa_{3}$ the opposite behavior is observed, signifying Kondo physics. The left inset is a gray scale plot of the differential conductance $d I / d V_{\text {sd }}$ versus $V_{g}$ and $V_{\text {sd }}$ (stability diagram) measured at $800 \mathrm{mK}\left(>T_{c}\right)$. It shows the familiar pattern of Coulomb diamonds (charging energy $E_{C} \approx 1.5 \mathrm{meV}$, level spacing $\Delta E \sim 1 \mathrm{meV}$ ) and confirms the presence of a high conductance Kondo ridge around zero bias through the diamond $\kappa_{1}$. The black dashed line shows $G$ versus $V_{g}$ measured at $300 \mathrm{mK}\left(<T_{c}\right)$. Instead of continuing their increase as expected for the Kondo effect (without superconductivity) the valley conductances $G_{v}$ of $\kappa_{1}-\kappa_{3}$ decrease below their values at $950 \mathrm{mK}$. This result is consistent with the findings of Ref. [3] and shows that the binding energy $\sim k_{B} T_{K}$ of the Kondo states $\kappa_{1}-\kappa_{3}$ is lower than the binding energy of the superconducting Cooper pairs $\Delta \approx 1.75 k_{B} T_{c}$. Here $k_{B}$ is Boltzmann's constant and the Kondo temperature $T_{K}$ is therefore smaller than $\Delta / k_{B} \approx 1.3 \mathrm{~K}$ in all three charge states $\kappa_{1}-\kappa_{3}$ [14]. The suppression of the Kondo state is confirmed by the disappearance of the Kondo ridge in the stability diagram measured at $300 \mathrm{mK}$ shown for $\kappa_{1}$ in the rightmost inset to Fig. 1(b).

The finite-bias peaks indicated by the arrows are observed throughout the stability diagram and appear symmetrically around $V_{\text {sd }}=0 \mathrm{~V}$ (see also Fig. 2). These are manifestations of the superconducting state of the contacts where peaks are expected at $\left|V_{\mathrm{sd}}\right|=2 \Delta / e$ when the den-

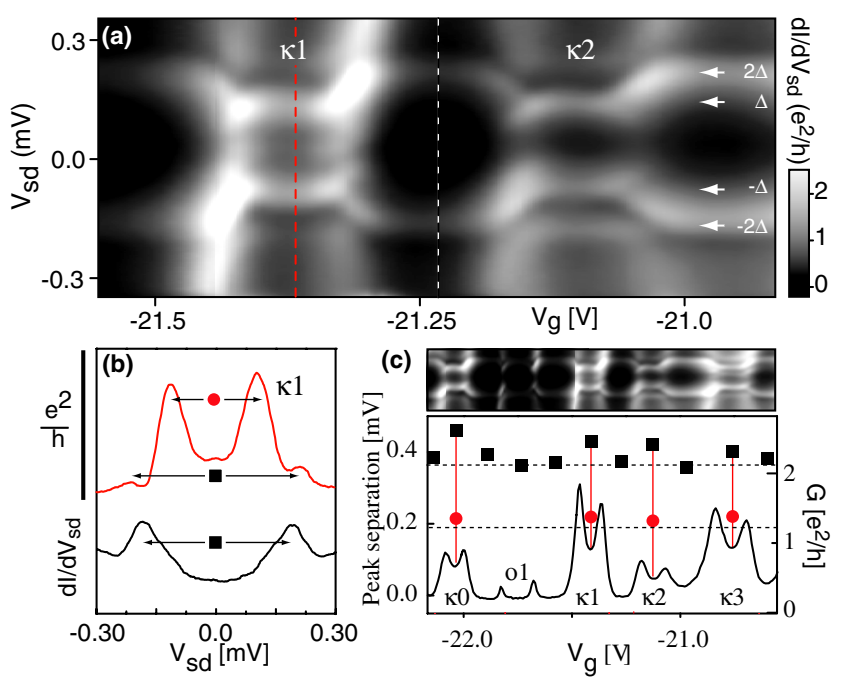

FIG. 2 (color online). (a) Stability diagram for the $V_{g}$ region of $\kappa_{1}$ and $\kappa_{2}$ and $\left|V_{\text {sd }}\right| \leq 0.35 \mathrm{mV}$ exhibiting a pronounced evenodd periodicity in the subgap structure. The approximate positions of the dominant peaks at $V_{\mathrm{sd}}= \pm \Delta / e, \pm 2 \Delta / e$ are indicated. (b) $d I / d V_{\text {sd }}$ vs $V_{\text {sd }}$ through the middle of two neighboring diamonds [lines in (a)] showing the enhanced peaks at $\left|V_{\mathrm{sd}}\right| \approx$ $\Delta / e$ for the Kondo diamond (curves offset for clarity). (c) Bias spectroscopy for a larger $V_{g}$ region showing the enhanced $\Delta$ peak for four Kondo diamonds. Main panel shows the linear conductance (rightmost axis) and the distance between $\pm \Delta$ and $\pm 2 \Delta$ subgap peaks in the middle of the diamonds [leftmost axis, symbols as in (b)]. Horizontal dashed lines indicate the position of $2 \Delta$ and $4 \Delta$.

sity of states at the gap edges line up as illustrated in Fig. 1(c). The stability diagram in Fig. 2(a) shows a detailed measurement of these low-bias features for the Coulomb diamonds of $\kappa_{1}$ and $\kappa_{2}$. Close to the degeneracy points of the Coulomb diamonds a complicated peak structure is observed, since in this region multiple Andreev reflections (MAR) occur resonantly through the gatevoltage dependent dot level $[11,15,16]$.

We restrict our discussion to the middle region of the Coulomb diamonds where the peak positions are largely gate independent and transport occurs by cotunneling between the two superconductors. In this case, MAR peaks are expected at $\left|V_{\text {sd }}\right|=2 \Delta / n e, n=1,2, \ldots$ with intensities determined mainly by the effective transparency of the device (defined below). The peaks at $\left|V_{\mathrm{sd}}\right|=\Delta / e(n=$ 2) result from the opening and closing of processes involving one and three Andreev reflections, respectively, as schematically shown in Fig. 1(d). The lower trace in Fig. 2(b) shows the differential conductance along the white dashed line in Fig. 2(a) through the middle of an even- $N$ diamond. As expected, peaks are observed at $\left|V_{\text {sd }}\right|=2 \Delta / e$ with fainter shoulders at $\left|V_{\text {sd }}\right|=\Delta / e$. However, as seen in Fig. 2(a) the SGS in the odd- $N$ diamonds of the suppressed Kondo ridges $\kappa_{1}$ and $\kappa_{2}$ is clearly modified. Unexpectedly, the peaks at $\left|V_{\mathrm{sd}}\right|=\Delta / e$ are more than 5 times larger than the peaks at $\left|V_{\mathrm{sd}}\right|=2 \Delta / e$, as emphasized 
by the upper trace in Fig. 2(b) through the middle of the $\kappa_{1}$ diamond. This contrasts the expectations for simple tunneling between the two superconductors, and the presence of the Kondo effect in the normal state points towards electron-electron correlations as the origin of the modified SGS [17].

Further support of the connection between the Kondo effect and the enhanced $\Delta$ peak is provided in Fig. 2(c): the lower panel shows the linear conductance (right axis) over a $V_{g}$ range of 5 odd- $N$ and 6 even- $N$ Coulomb valleys. In the three odd- $N$ valleys $\kappa_{1}-\kappa_{3}$ from Fig. 1(b) and an additional one, $\kappa_{0}$, the Kondo effect was observed in the normal state, and as seen in the upper panel, the enhanced $\Delta$ peak is observed in the SGS of each Coulomb diamond. In the remaining diamonds, including $o 1$ with odd- $N$ (which did not show the Kondo effect in the normal state), the conventional SGS is observed. Thus, the effect is connected to Kondo correlations rather than to the number of electrons on the dot. In the lower panel of Fig. 2(c) the separations between the $-2 \Delta$ and $+2 \Delta$ peaks (squares) and between the $-\Delta$ and $+\Delta$ peaks (circles) are extracted for each Coulomb valley (left axis). The separations of the $\pm 2 \Delta$ peaks depend slightly on $N$ and are increased in the $\kappa$ valleys with respect to the even- $N$ values; however, the $\Delta$ peaks in the $\kappa$ valleys always appear at exactly half the separation of the corresponding $2 \Delta$ peaks.

To investigate further the origin of the $\Delta$ peak we study the temperature dependence of the SGS. Figure 3(a) shows $d I / d V_{\text {sd }}$ versus $V_{\text {sd }}$ through the middle of $\kappa_{1}$ for temperatures $1700 \mathrm{mK}-310 \mathrm{mK}$. Upon lowering the temperature,
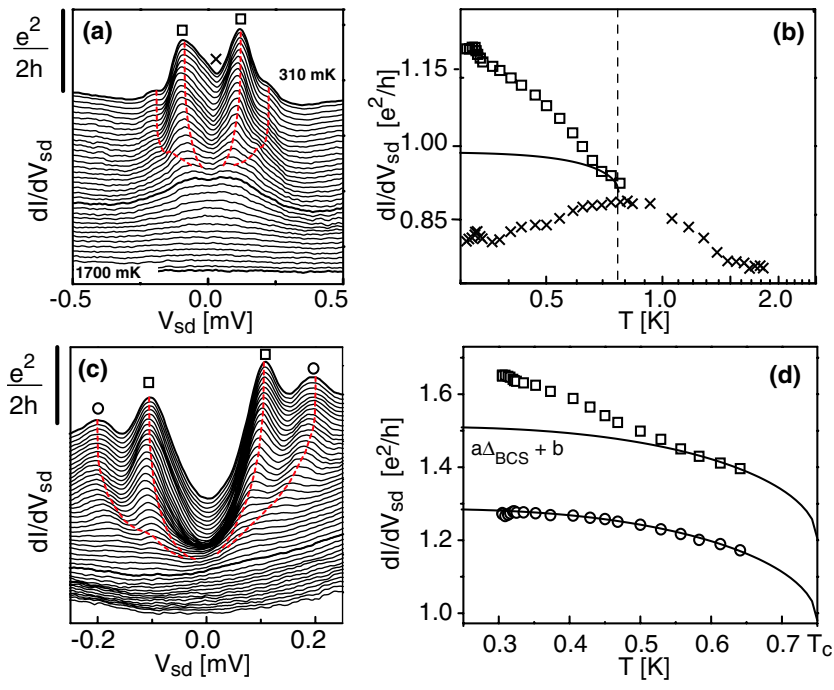

FIG. 3 (color online). (a) $d I / d V_{\text {sd }}$ vs $V_{\text {sd }}$ through $\kappa_{1}$ for different temperatures $310 \mathrm{mK}-1700 \mathrm{mK}$ (offset for clarity). Bold trace shows the Kondo peak at $T=800 \mathrm{mK}$ and the formation of the $\Delta / e$ peak can be followed. Symbols correspond to traces in (b) showing the suppression of the valley conductance (crosses) and the continuing increase of the average heights of the $\Delta$ peaks below $T_{c}$ (squares). Solid line shows a fit to the gap function $\Delta_{\mathrm{BCS}}(T)$. (c), (d) As in (a), (b) from another device. the initial formation of the Kondo peak is observed for $T>$ $T_{c}$, followed by the formation of the subgap peaks at $\left|V_{\mathrm{sd}}\right|=\Delta / e$ with shoulders at $\left|V_{\mathrm{sd}}\right|=2 \Delta / e$ for $T<T_{c}$. The peak positions follow the expected temperature dependence of the BCS gap $\Delta_{\mathrm{BCS}}(T)$ (red lines). To allow for a better comparison of the evolution of the $\Delta$ and $2 \Delta$ peaks we include in Fig. 3(c) a similar measurement performed for a suppressed Kondo ridge in a different device where the four peaks can be clearly distinguished. In Figs. 3(b) and 3(d) the temperature dependencies of the average peak heights and the valley conductances have been extracted. In both cases the $\Delta$ peaks increase with roughly constant slope, whereas the $2 \Delta$ peaks of the measurement in Fig. 3(c) saturate below $\sim 0.5 \mathrm{~K}$. In the absence of interactions the temperature dependence is governed by the gap, and we expect the measured peak heights to be proportional to $\Delta_{\mathrm{BCS}}(T)$ [18]. The solid lines in Figs. 3(b) and 3(d) show such fits and $\Delta_{\mathrm{BCS}}(T)$ indeed describes the behavior of the $2 \Delta$ peak in Fig. 3(d). For the enhanced $\Delta$ peaks, however, the continuing strengthening is not captured, thereby adding further evidence to the importance of correlations for the origin of these peaks.

In the middle of the Coulomb diamonds, charge fluctuations are strongly suppressed and electrons traverse the dot via cotunneling processes with a tunneling amplitude of the order of $J_{L R} \sim t_{L}^{*} t_{R} / E_{C}$, where $t_{L, R}$ are the tunneling amplitudes from the dot to the two leads. This means that the even occupied dot can be viewed as an effective superconducting single-mode junction with transparency $\alpha \sim$ $\left(\nu_{F} J_{L R}\right)^{2} \ll 1$, which has been studied in Ref. [19] $\left(\nu_{F}\right.$ denotes the density of states at the Fermi levels). In singlemode junctions with low transparency, the quasiparticle tunneling conductance peak at $\left|V_{\mathrm{sd}}\right|=2 \Delta / e$ dominates over the subgap peaks, as observed in our even Coulomb diamonds. In the case of odd occupation, however, transport occurs via exchange (co)tunneling which gives rise to a Kondo-enhanced transparency of the order of $1 / \ln ^{2}\left(\Delta / k_{B} T_{K}\right)$. Since the leading subgap peak at $V_{\text {sd }}=$ $\Delta / e$ can exceed the $2 \Delta$ peak for moderate values of the transparency [19], our observations are consistent with a Kondo-enhanced transparency of the junction. This argument is valid only for $\Delta \gg k_{B} T_{K}$, but clearly the spin-full dot holds the premise for a Kondo-enhanced transparency, which provides a simple understanding of the $\Delta$ peak dominating the $2 \Delta$ peak in the odd diamonds which supported a Kondo resonance for temperatures above $T_{C}$.

The logarithmic enhancement of the transparency can be established from a poor man's scaling analysis [20] of the Kondo model with superconducting leads and $\Delta \gg k_{B} T_{K}$. The exchange coupling, $\nu_{F} J_{L R}$, grows stronger as the conduction electron bandwidth, $D$, is reduced, and terminates at $1 / \ln \left(\Delta / k_{B} T_{K}\right)$ when $D$ reaches $\Delta$. Interestingly, the scaling also generates a new anomalous operator independent of the impurity spin. The effective low-energy (time-dependent) Hamiltonian thus contains an Andreev- 
tunneling term, $A_{T}\left[e^{2 i\left(\mu_{L}-\mu_{R}\right) t} \sum_{k^{\prime}, k} c_{L k^{\prime} \uparrow}^{\dagger} c_{L k \downarrow}^{\dagger}+(L \leftrightarrow R)\right]+$ H.c., where $\mu_{L, R}$ denotes the chemical potentials and the explicit time dependence from the applied bias has been gauged into a phase factor on the electron operators. The current is readily calculated to second order in $A_{T}$ and sets in with a step at $V=\Delta$. Schematically, the coupled scaling equations for $J$ and $A_{T}$ take the form

$$
\begin{aligned}
\frac{d A_{T}}{d \ln D} & =-\frac{3}{4} \frac{\Delta}{D} J^{2}-4 \frac{\Delta}{D} A_{T}^{2}, \\
\frac{d J}{d \ln D} & =-J^{2}+2 \frac{\Delta}{D} J A_{T},
\end{aligned}
$$

leaving out all lead indices $(L, R)$ and step functions determining the energy scales beyond which the various terms no longer contribute to the flow. For $D \gg \Delta$, only $J$ grows logarithmically whereas the flow of $A_{T}$ is prohibited by extra factors of $\Delta / D$ deriving from the virtual propagation of a Cooper pair near the band edge. For $D \ll \Delta$, however, these factors of $\Delta / D$ would rather enhance the flow and lead to a divergence of $A_{T}$. We postpone the full analysis of these scaling equations to a future publication [21], and we merely note here that the alignment of the Fermi level with the gap edge for $\left|V_{\mathrm{sd}}\right|=\Delta / e$ might permit the flow to continue to strong coupling, which in turn may lead to the anomalous temperature dependence which we observe for the $\Delta$-peak height. Further analysis of the finite-bias scaling equations will clarify this issue. Finally, we note that the Andreev-tunneling operator is also generated in the even diamonds by simple potential scattering, but in this case the $J^{2}$ term driving the enhancement of the transparency is missing.

In summary, we have discovered a pronounced alternation of the strength of the leading subgap conductance peak between even and odd occupied quantum dots coupled to superconducting leads. We have observed this effect in 15 suppressed Kondo ridges in two different devices and we ascribe the enhancement of the $\Delta$ peaks for odd occupations to a Kondo-enhanced Andreev-tunneling amplitude. Furthermore, we have found that, unlike the $2 \Delta$ peak, the $\Delta$-peak height does not saturate with $\Delta(T)$ when lowering the temperature.

This work was supported by the EC FP6 funding (Project No. FP6-IST-003673) and the Danish Agency for Science, Technology, and Innovation.

Note added. - Recently, we have become aware of an independent, parallel study of the above phenomenon in a different materials system, carbon nanotubes, by A. Eichler et al. [22].

[1] D. Goldhaber-Gordon et al., Nature (London) 391, 156 (1998).

[2] L. P. Kouwenhoven and L. I. Glazman, Phys. World 14, 33 (2001).

[3] M.R. Buitelaar, T. Nussbaumer, and C. Schönenberger, Phys. Rev. Lett. 89, 256801 (2002).

[4] Y. J. Doh et al., Science 309, 272 (2005).

[5] J. A. van Dam et al., Nature (London) 442, 667 (2006).

[6] H. I. Jørgensen et al., Phys. Rev. Lett. 96, 207003 (2006).

[7] P. Jarillo-Herrero, J. A. van Dam, and L. P. Kouwenhoven, Nature (London) 439, 953 (2006).

[8] A. F. Andreev, Sov. Phys. JETP 19, 1228 (1964).

[9] T. M. Klapwijk, G.E. Blonder, and M. Tinkham, Physica $\mathrm{B}+\mathrm{C}$ (Amsterdam) 110, 1657 (1982); M. Octavio, M. Tinkham, G.E. Blonder, and T.M. Klapwijk, Phys. Rev. B 27, 6739 (1983); K. Flensberg, J. B. Hansen, and M. Octavio, Phys. Rev. B 38, 8707 (1988).

[10] E. Scheer et al., Phys. Rev. Lett. 78, 3535 (1997).

[11] M. R. Buitelaar et al., Phys. Rev. Lett. 91, 057005 (2003).

[12] M. Aagesen et al. (to be published).

[13] T. S. Jespersen et al., Phys. Rev. B 74, 233304 (2006).

[14] In agreement with Ref. [3] we have also observed Kondo ridges that survive the transition to superconducting contacts and are further enhanced by the superconductor as expected for $T_{K}>\Delta / k_{B}$.

[15] A.L. Yeyati, J.C. Cuevas, A. López-Dávalos, and A. Martin-Rodero, Phys. Rev. B 55, R6137 (1997).

[16] G. Johansson, E.N. Bratus, V.S. Shumeiko, and G. Wendin, Phys. Rev. B 60, 1382 (1999).

[17] We note that a similar enhanced $\Delta / e$ peak in the SGS of a carbon nanotube QD is evident in Refs. [3,11] and in K. Grove-Rasmussen, H. I Jørgensen, and P. E. Lindelof, New J. Phys. 9, 124 (2007); Proceedings of the International Symposium on Mesoscopic Superconductivity and Spintronics 2006 (World Scientific, Singapore, 2007).

[18] E. L. Wolf, Principles of Electron Tunneling Spectroscopy (Oxford University, New York, 1985), p. 113.

[19] D. Averin and A. Bardas, Phys. Rev. Lett. 75, 1831 (1995); E. N. Bratus, V. S. Shumeiko, and G. Wendin, Phys. Rev. Lett. 74, 2110 (1995); J. C. Cuevas, A. Martin-Rodero, and A. L. Yeyati, Phys. Rev. B 54, 7366 (1996).

[20] P. W. Anderson, J. Phys. C 3, 2436 (1970).

[21] J. Paaske, B. M. Andersen, and K. Flensberg (unpublished).

[22] A. Eichler et al., Phys. Rev. Lett. 99, 126602 (2007). 